\title{
Combining Percutaneous Ultrasound-Guided Hematoma Aspiration and Compression Repair to Treat Femoral Artery Pseudoaneurysm after Cardiac Catheterization
}

\author{
Gang Chen, ${ }^{1, *}$ MD, Lingmin Wu, ${ }^{1, *}$ MD, Lihui Zheng, ${ }^{1}$ MD, Ligang Ding, ${ }^{1}$ MD, Tom Wong, ${ }^{2}$ MD, \\ Shu Zhang, ${ }^{1}$ MD and Yan Yao, ${ }^{1}$ MD
}

\begin{abstract}
Summary
This study aimed to prospectively evaluate the safety and efficacy of a new developed method that uses percutaneous ultrasound-guided hematoma aspiration followed by targeted localized manual compression for treatment of femoral artery pseudoaneurysm after cardiac catheterization, which obviates thrombin use, surgery, and long-time compression.

From January 2007 to July 2014,32 patients ( 17 women; mean age, $55.3 \pm 11.5$ years) out of 8,725 consecutive cases undergoing cardiac catheterization via femoral access developed one pseudoaneurysm each ranging in size from $21 \times 11 \mathrm{~mm}$ to $72 \times 39 \mathrm{~mm}$. Under ultrasound guidance, blood within the pseudoaneurysm was aspirated percutaneously using an 18 -gauge needle, while the pseudoaneurysm neck and a nearby site over the pseudoaneurysm were manually compressed for $15 \mathrm{~min}$. All patients underwent repeat ultrasound examination 24 hours later.

Of the 32 pseudoaneurysms, 31 were successfully occluded, and 1 recurred in a patient with coexisting arteriovenous fistula, yielding an overall success rate of $96.9 \%$ (31/32). No further recurrence or procedure related complications were observed. The treatment approach is unlike open surgical repair with hematoma evacuation and arterial defect suturing, in that it entails hematoma aspiration and feeding flow blockage at the pseudoaneurismal neck.

In this preliminary experience, combining percutaneous ultrasound-guided hematoma aspiration and manual compression appears safe and effective in treating femoral artery pseudoaneurysms after catheterization and avoids thrombin use, long-time compression, and surgery.
\end{abstract}

Key words: Femoral pseudoaneurysm, Treatment, Complication

(Int Heart J 2018; 59: 333-338)

I

atrogenic femoral pseudoaneurysm post-catheterization, which occurs in up to $0.5 \%$ and $8 \%$ of diagnostic and interventional cardiac catheterization cases, respectively, may cause persistent groin pain and swelling, compression neuropathy, limb ischemia, and skin necrosis, and even undergo sudden rupture..$^{-4)}$

Options for intervention of $>2 \mathrm{~cm}$ and/or symptomatic post-catheterization femoral artery pseudoaneurysms include surgical repair, with a $20 \%$ rate of complications including major bleeding, infection and death, ${ }^{5)}$ or the now favored noninvasive ultrasound-guided compression repair, with $74 \%$ success rate but requiring long and discomforting manual compression; ${ }^{6,7)}$ and thrombin injection, with $\geq$ $90 \%$ overall success rate but with potential serious complications. ${ }^{8-10)}$ We therefore evaluated the safety and efficacy of a new ultrasound-guided treatment, which obvi-

ates thrombin use, long-time compression and surgery.

\section{Methods}

Study population: From January 2007 to July 2014, a total of 8,725 radiofrequency catheter ablation and percutaneous coronary intervention procedures were performed in our hospital. Vascular ultrasound examination of the femoral artery was performed after catheterization in patients with hematoma, suspicious swelling, vascular murmurs, or any other clinical abnormality of the puncture site. After the exclusion of one patient who refused to provide informed consent and preferred an ultrasound-guided compression approach, the study enrolled 32 consecutive patients ( 17 women; mean age, $55.3 \pm 11.5$ years) with evidence of an "uncomplicated" pseudoaneurysm (i.e., with-

From the ${ }^{1}$ State Key Laboratory of Cardiovascular Disease, Fuwai Hospital, National Center for Cardiovascular Diseases, Chinese Academy of Medical Sciences and Peking Union Medical College, Beijing, China and ${ }^{2}$ Heart Rhythm Centre, Biomedical Research Unit, Royal Brompton and Harefield NHS Foundation Trust, London, United Kingdom.

*These authors contributed equally to this work.

Address for correspondence: Yan Yao, MD, State Key Laboratory of Cardiovascular Disease, Fuwai Hospital and Cardiovascular Institute, 167 Beilishi Road, Xicheng, Beijing 100037, China. E-mail: ianyao@263.net.cn

Received for publication January 15, 2017. Revised and accepted May 9, 2017.

Released in advance online on J-STAGE March 20, 2018.

doi: 10.1536/ihj.17-026

All rights reserved by the International Heart Journal Association. 


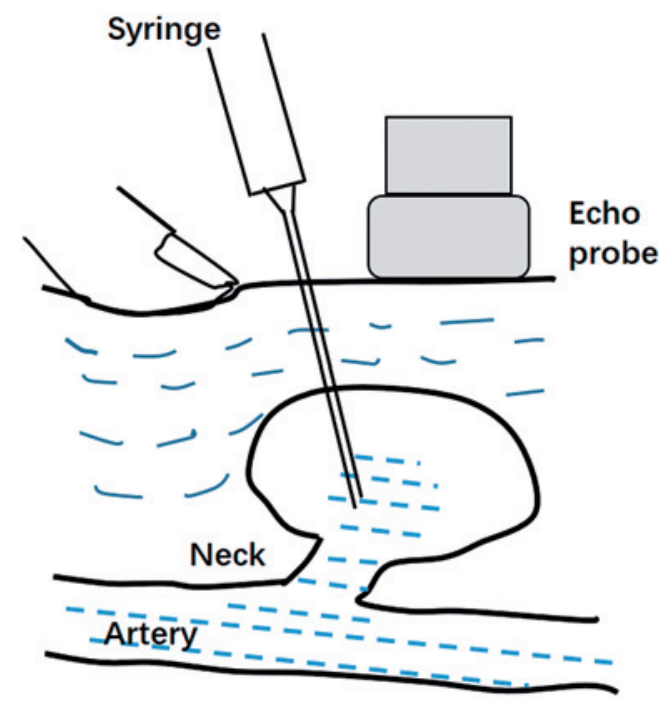

Figure 1. Schematic diagram of percutaneous ultrasound-guided hematoma aspiration followed by targeted localized manual compression. Schematic diagram shows a spinal needle attached to a $20 \mathrm{~mL}$ syringe puncturing into the liquid center of a pseudoaneurysm. After carefully locating under color Doppler ultrasound guidance the position of the neck $(\mathrm{N})$ and the center of the pseudoaneurysm $(\mathrm{P})$, marks are made on the overlying skin directly over them (arrows denote those for the neck). A doctor uses the needle to puncture and aspirate. As soon as the needle tip is inserted into the liquid center of the aneurysm, red arterial blood gushes into the syringe through the hub of the needle. At the same time, an assistant immediately compresses the position indicated by the marker (arrow) directly over the neck of the pseudoaneurysm (N) to completely arrest flow into the aneurysm from supply artery (A). The doctor continuously aspirates the hematoma until no more blood can be drawn.

out local skin infection or related distal limb arterial stenosis).

Procedure: Informed written consent was obtained from all patients, and this study was approved by the Ethics Committee of our hospital, and clinical investigations are conducted according to the principles expressed in the Declaration of Helsinki. All patients and alternative therapies (such as ultrasound-guided compression repair (UGCR) versus observation) were discussed. All 32 patients had undergone catheterization 1 to 5 days (mean 1.9 \pm 1.3 days) prior to their enrollment. Catheter sheath size ranged from $6 \mathrm{~F}$ to $9 \mathrm{~F}$.

The patients were examined using an ultrasound system (GE LOGIQ S8 with a 2-8 MHz linear array transducer; or SIEMENS Acuson Antares with a 4-9 MHz linear probe). The width of the pseudoaneurismal neck and the number and size of pseudoaneurismal chambers were documented. The supply artery was identified and recorded. The arterial vasculature of the affected extremity was also examined.

The skin overlying the pseudoaneurysm was prepped with iodophor and was covered with sterile drapes. The positions of the pseudoaneurismal neck and center were identified by color Doppler ultrasound and marked on the overlying skin. The echo probe within a sterile pouch was positioned directly over the pseudoaneurysm for ultrasound guidance.
The following two steps were performed (Figure 1): Step 1 Using the puncture technique previously described in ultrasound-guided thrombin injection (UGTI), ${ }^{8)}$ the pseudoaneurismal hematoma was punctured under subcutaneous anesthesia ( $0.5 \%$ lidocaine) with an 18 -gauge spinal needle attached to a $20 \mathrm{~mL}$ syringe and using negative pressure. Puncture is done before compression because shape and relative positions of pseudoaneurysm, supply artery, and subcutaneous tissue are preserved without outside pressure easing needle insertion into the aneurysm center. As soon as the needle tip reached the liquid center of the aneurysm and aspiration was achieved, an assistant compressed the marked neck position to block arterial flow into the aneurysm, while the physician kept aspirating the hematoma until no more blood could be drawn. There was nothing injected into the pseudoaneurysm during the whole procedure. Puncture was done before compression because the shape and relative positions of pseudoaneurysm, supply artery, and subcutaneous tissue were preserved without outside pressure easing needle insertion into the aneurysm center.

Step 2 After needle withdrawal, the assistant continued pseudoaneurismal neck compression for $15 \mathrm{~min}$, while the physician compressed a nearby site over the pseudoaneurysm to prevent potential leakage. The latter compression is as in UGCR, ${ }^{6}$ ) except that compression pressure is targeted at the pseudoaneurismal neck.

A pressure dressing then was applied during 12 hours of bed rest with the affected leg of the patient frequently massaged to prevent deep vein thrombosis.

To rule out recurrent hematoma/pseudoaneurysm, ultrasound examination was repeated in all subjects 24 hours after release of the pressure dressing. Success was defined as completely thrombosed pseudoaneurysm. The procedure for puncturing and aspiration are shown in Figure 2. When complete thrombosis of pseudoaneurysm was confirmed, the patient then can be discharged if other clinical circumstance permitted. If active flow persisted toward the aneurysm, a second session of ultrasound-guided hematoma aspiration and manual compression was performed. All subjects were followed as outpatients for at least 3 to 6 months after the procedure.

Statistical analysis: Continuous variables are presented as mean \pm standard deviation, range, and minimum and maximum values. The Student's $t$-test was used to compare the difference of age between female and male patients. A $P$ value of $<0.05$ was considered statistically significant.

\section{Results}

Baseline clinical characteristics of 32 patients were shown in Table I. There was a tendency that the mean age of female patients was higher than that of male patients; however, the difference between them was not statistically significant $(P=0.055)$. All 32 patients studied had one post-catheterization pseudoaneurysm: 29 were monolobulated and 3 were bilobulated. Of the 32 patients, $100 \%$ were on anticoagulant or at least one antiplatelet before aspiration. Pseudoaneurysms were detected $1.9 \pm 1.3$ days (1 to 5 days) after catheterization, with sizes ranging from 

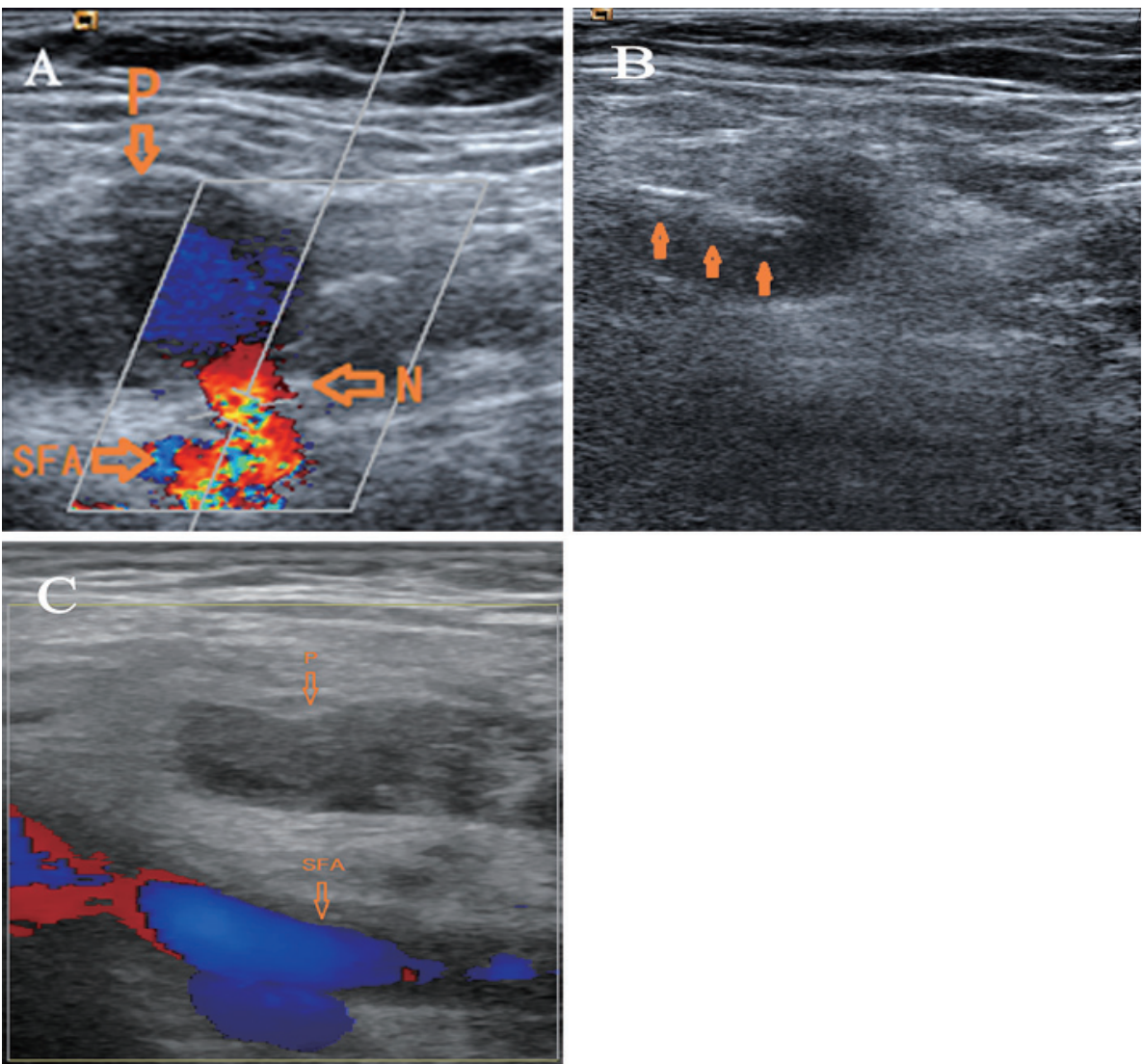

Figure 2. Procedure for puncture and aspiration. A: The femoral artery pseudoaneurysm. (P) originating from the superficial femoral artery (SFA) and neck between them. B: Procedure for puncture and aspiration. The needle is inserted into the liquid center of the pseudoaneurysm (arrow) under ultrasound guidance with color flow turned off. C: Complete thrombosis of the pseudoaneurysm 2 days after pressure dressing release.

$21 \times 11 \mathrm{~mm}$ to $72 \times 39 \mathrm{~mm}($ mean $[38.3 \pm 13.8] \times[19.2$ $\pm 8.2] \mathrm{mm}$ ), that is, all larger than $2 \mathrm{~cm}$ (Table II). Patients experienced pseudoaneurysm-related symptoms including groin pain and/or swelling.

Of the 32 pseudoaneurysms, 29 (90.6\%) were successfully occluded after the first attempt; and $2(6.3 \%)$ early cases required a second attempt to achieve complete thrombosis, yielding an overall success rate of $96.9 \%$. The only failure was in a patient with a coexisting arteriovenous $(\mathrm{AV})$ fistula.

Among four patients with coexisting AV fistulae, one AV fistulae of one patient was occluded at the same time as the pseudoaneurysm achieved full thrombosis after aspiration and compression, two patients who had a successful aspiration and compression procedures to treat pseudoaneurysms have the AV fistulae left, and the fourth patient was the patient whose procedure was not successful, where the pseudoaneurysm was not fully occluded (reduced from $50 \times 10$ to $14 \times 10 \mathrm{~mm}$ ) and the AV fistulae left.

Complete pseudoaneurysm thrombosis was achieved in all 18 of $32(56 \%)$ patients on anticoagulant therapy. These 18 patients underwent coronary interventions or atrial fibrillation/atrial flutter ablation. Patients received low molecular weight heparin in a body weight-adjusted dose (enoxaparin $1 \mathrm{mg} / \mathrm{kg}$, twice daily), aspirin (100 mg, once daily), and clopidogrel (75 $\mathrm{mg}$, once daily) after coronary interventions, or received both low molecular weight heparin in a body weight-adjusted dose (enoxaparin $1 \mathrm{mg} / \mathrm{kg}$, twice daily) and/or warfarin (3 $\mathrm{mg}$, once daily) after atrial fibrillation/atrial flutter ablation. Low molecular weight heparin and warfarin were discontinued on the procedure day and resumed immediately upon pseudoaneurysm obliteration confirmation. No patient required reversal of anticoagulation with vitamin $\mathrm{K}$ or other antagonists.

There was no thrombin injection-related complication such as arterial thrombosis and allergic reaction due to avoidance of using thrombin in the whole procedure. In the procedure, aspiration of the hematoma decreases local tension on the groin thereby significantly relieving associated pain and swelling. Therefore, this method was well tolerated by all patients with groin pain and swelling reduction. Doppler ultrasound scan was repeated 24 hours after the first attempt for all patients, and in the two subjects requiring a second attempt, it was repeated at 1 to 3 days after the second treatment attempt, showing no hematoma recurrence. Patients with pseudoaneurysm treated with the described method stayed $1.09 \pm 0.3$ day more than the patients without pseudoaneurysm. 
During 3-6 months follow-up, there was no late recurrence or procedural or later complications. All three AV fistulae were occluded in 3-6 months later. The pseudoaneurysm of the unsuccessful patient was found to be fully obliterated spontaneously at 3 months follow-up.

\section{Discussion}

In the present study of 32 patients, a combination approach comprising percutaneous ultrasound-guided hematoma aspiration and UGCR's targeted manual compression for the treatment of femoral artery pseudoaneurysm after cardiac catheterization appeared technically feasible; safe without complications; and efficacious, with high primary $(90.6 \%)$ and overall $(96.9 \%)$ success rates and groin pain and swelling reduction.

Table I. Basic Clinical Characteristics of the Study Cohort $(n=32)$

\begin{tabular}{|c|c|}
\hline Variable & \\
\hline Male & $15(46.9 \%)$ \\
\hline Age, years & $55.3 \pm 11.5$ \\
\hline Female & $58.1 \pm 9.3$ \\
\hline Male & $52.1 \pm 13.1$ \\
\hline Body mass index, $\mathrm{kg} / \mathrm{m}^{2}$ & $24.1 \pm 2.7$ \\
\hline \multicolumn{2}{|l|}{ Comorbidity } \\
\hline Hypertension & $8(1.9 \%)$ \\
\hline Coronary artery disease & $11(34 \%)$ \\
\hline Diabetes mellitus & $6(19 \%)$ \\
\hline Rheumatic valvular disease & $3(9 \%)$ \\
\hline Anticoagulation therapy before aspiration & $18(56 \%)$ \\
\hline LMWH + warfarin & $8(25 \%)$ \\
\hline LMWH + aspirin + clopidogrel & $7(22 \%)$ \\
\hline Warfarin & $3(9 \%)$ \\
\hline Antiplatelet therapy before aspiration & $14(44 \%)$ \\
\hline \multicolumn{2}{|l|}{ Procedures that the aneurysms developed after } \\
\hline Radiofrequency catheter ablation & $23(72 \%)$ \\
\hline Percutaneous coronary intervention & $9(28 \%)$ \\
\hline \multicolumn{2}{|l|}{ Sheath size, Fr } \\
\hline $6 \mathrm{~F}, n$ & $8(25 \%)$ \\
\hline $7 \mathrm{~F}, n$ & $12(38 \%)$ \\
\hline $8 \mathrm{~F}, n$ & $11(34 \%)$ \\
\hline $9 \mathrm{~F}, n$ & $1(3 \%)$ \\
\hline \multicolumn{2}{|l|}{ Catheter access } \\
\hline Artery access & $21(66 \%)$ \\
\hline Vein access & $11(34 \%)$ \\
\hline
\end{tabular}

LMWH indicates low molecular weight heparin.
Small pseudoaneurysms can be treated conservatively; however, catheter-related femoral artery pseudoaneurysms that exceed $2 \mathrm{~cm}$ in diameter and/or are symptomatic should be treated more aggressively to alleviate patient's persistent groin pain and to minimize occurrence of related complications such as compression neuropathy, limb ischemia, or the most feared one of sudden rupture. ${ }^{7-10)}$

Treatment of femoral artery pseudoaneurysm after cardiac catheterization remains challenging, and current choices have limitations. ${ }^{11)}$ Open surgical repair has a high $(\sim 20 \%)$ rate of complications including bleeding $(7.4 \%)$, neuralgia $(5.2 \%)$, and death $(2.1 \%){ }^{5,12)}$ The use of thrombin to promote clot formation within the pseudoaneurysm is a more recent development and although effective, ${ }^{8,13)}$ serious complications such as arterial thrombosis and allergic reactions have been reported. ${ }^{14-16)}$ Although in some studies use of autologous thrombin or recombinant human thrombin to treat pseudoaneurysms helped to avoid risk of anaphylaxis associated with use of bovine thrombin, ${ }^{17,18)}$ it did not reduce the risk of arterial thrombosis and may increase cost.

UGCR, which was introduced by Fellmeth and colleagues in 1991 and includes ultrasound-guided compression to block flow at the pseudoaneurismal neck, ${ }^{6}$ has proven safe and cost-effective with a success rate of $74 \%$, which is not as high as expected. Also, UGCR is often time-consuming, painful and even intolerable for patients despite intravenous conscious sedation. ${ }^{1,8)}$ Why? We posited that radial dispersion of the compression pressure by the pocket of uncoagulated blood collection in the pseudoaneurysm reduces the efficacy of manual compression, and compression pressure significantly increases pseudoaneurysm tension that translates into significantly increased pain. On the basis of this hypothesis, aspiration of the hematoma would greatly reduce pseudoaneurysm tension while significantly ameliorating patient's discomfort thereby enhancing tolerance to subsequent manual compression.

The treatment approach described here is not unlike open surgical repair with hematoma evacuation and arterial defect suturing, in that it entails hematoma aspiration and feeding flow blockage at the pseudoaneurismal neck. Ultrasound-guided aspiration of the uncoagulated blood at the pseudoaneurysm center decreases pseudoaneurysm tension allowing compression pressure to be applied di-

Table II. Ultrasound Characteristics of Pseudoaneurysms

\begin{tabular}{lc}
\multicolumn{2}{c}{ Variable } \\
\hline Pseudoaneurysm size & \\
$\quad$ Length, mm & $38.3 \pm 13.8(17-75)$ \\
Width, mm & $19.2 \pm 8.2(8-39)$ \\
Neck width, mm & $1.9 \pm 0.58(1-3)$ \\
Pseudoaneurysms & \\
$\quad$ Pseudoaneurysms alone & $28(87.5 \%)$ \\
Pseudoaneurysms coexisting with arteriovenous fistula & $4(12.5 \%)$ \\
Supply artery & $16(50 \%)$ \\
Common femoral artery & $14(44 \%)$ \\
Superficial femoral artery & $2(6 \%)$ \\
Deep femoral artery &
\end{tabular}


rectly to the pseudoaneurismal neck to arrest blood inflow and relieving pseudoaneurysm-related swelling and pain, with stasis of the small amount of residual blood facilitating thrombus formation within the pseudoaneurysm. The latter features might underlie the technique's apparent success, even in patients on anticoagulation therapy. Complete pseudoaneurysm thrombosis was achieved in all 18 of $32(56 \%)$ patients on anticoagulant therapy, with low molecular weight heparin and warfarin discontinued on procedure day and resumed upon pseudoaneurysm obliteration confirmation and without need reversal of anticoagulation with vitamin $\mathrm{K}$ or other antagonists.

Avoidance of thrombin use and surgical repair may be the one of major features of the present treatment method. Lack of thrombin use not only avoids the risks of arterial thrombosis or allergic reactions but also thrombin cost. Avoidance of surgical repair, high success rate, pain and swelling amelioration, need for only 15 min compression, and no need for conscious sedation might improve the patient experience and reduce doctor involvement.

In addition, because the neck of the pseudoaneurysm was often relatively short and small, the manual pressure was actually exerted to the small area around the neck including artery proximal to pseudoaneurysm, neck, and the partial body mass of pseudoaneurysm near the artery. The advantage of our method is that the liquid blood of pseudoaneurysm was nearly empty after aspiration; thus, the strength could be exerted to this area easily and block the communication between the artery and the aneurysm without tension dispersion.

Limitations: The present study has several limitations. Firstly, the sample size was relatively small as in several similar early studies, ${ }^{6,8,17)}$ and the population studied was relatively young (mean of 55.3 years) and developed mostly monolobulated pseudoaneurysms. Secondly, it is plausible that a higher body mass index may make it more difficult to access followed by compression of the hematoma even with ultrasound guidance. However, in our current cohort, there is only one patient who failed to be treated; thus, we could not draw a conclusion that the failure was related to an increase of body mass index. It was worth noting that patients coexisting with local skin infection and related distal limb arterial stenosis were not suitable for this treatment. However, it might be too early to define the unsuitable clinical scenarios.

Larger and comparative clinical trials are warranted including: (1) clinical and cost-effectiveness comparisons with other techniques such as ultrasound-guided compression repair or thrombin injection; (2) greater proportions of more challenging clinical presentations such as older patients with peripheral artery disease and calcified vessels undergoing percutaneous coronary interventions and developing larger or multilobulated pseudoaneurysms, patients on anticoagulation therapy, and those with coexisting pseudoaneurysm with AV fistula; and (3) any restrictions to the cases treated with this method, such as a very large neck or inability to identify a legible neck.

\section{Conclusion}

In 32 patients evaluated, percutaneous ultrasound- guided hematoma aspiration followed by targeted localized manual compression appears safe and effective for the treatment of femoral artery pseudoaneurysms after cardiac catheterization with a success rate of $96.9 \%$ and avoidance of thrombin use, surgical repair, and long-time compression.

\section{Disclosures}

Conflicts of interest: None.

\section{References}

1. Schneider C, Malisius R, Küchler R, et al. A prospective study on ultrasound-guided percutaneous thrombin injection for treatment of iatrogenic post-catheterisation femoral pseudoaneurysms. Int J Cardiol 2009; 131: 356-61.

2. Kassem HH, Elmahdy MF, Ewis EB, Mahdy SG. Incidence and predictors of post-catheterization femoral artery pseudoaneurysms. Egypt Heart J 2013; 65: 213-21.

3. Gayed M, Yadak N, Qamhia W, Daralammouri Y, Ohlow MA. Comorbidities and complications in nonagenarians undergoing coronary angiography and intervention. Int Heart J 2017; 58: 180-4.

4. Kochman J, Rymuza B, Huczek Z, et al. Incidence, predictors and impact of severe periprocedural bleeding according to VARC-2 criteria on 1-year clinical outcomes in patients after transcatheter aortic valve implantation. Int Heart J 2016; 57: 3540.

5. Lumsden AB, Miller JM, Kosinski AS, et al. A prospective evaluation of surgically treated groin complications following percutaneous cardiac procedures. Am Surg 1994; 60: 132-7.

6. Fellmeth BD, Roberts AC, Bookstein JJ, et al. Postangiographic femoral artery injuries: nonsurgical repair with US-guided compression. Radiology 1991; 178: 671-5.

7. Schaub F, Theiss W, Busch R, Heinz M, Paschalidis M, Schömig A. Management of 219 consecutive cases of postcatheterization pseudoaneurysm. J Am Coll Cardiol 1997; 30: 670-5.

8. Kang SS, Labropoulos N, Mansour MA, Baker WH. Percutaneous ultrasound guided thrombin injection: a new method for treating postcatheterization femoral pseudoaneurysms. J Vasc Surg 1998; 27: 1032-8.

9. Kent KC, McArdle CR, Kennedy B, Baim DS, Anninos E, Skillman JJ. A prospective study of the clinical outcome of femoral pseudoaneurysms and arteriovenous fistulas induced by arterial puncture. J Vasc Surg 1993; 17: 125-31; discussion 1313.

10. Hirsch AT, Haskal ZJ, Hertzer NR, et al. ACC/AHA 2005 guidelines for the management of patients with peripheral arterial disease (lower extremity, renal, mesenteric, and abdominal aortic): executive summary a collaborative report from the American Association for Vascular Surgery/Society for Vascular Surgery, Society for Cardiovascular Angiography and Interventions, Society for Vascular Medicine and Biology, Society of Interventional Radiology, and the ACC/AHA Task Force on Practice Guidelines (Writing Committee to Develop Guidelines for the Management of Patients With Peripheral Arterial Disease) endorsed by the American Association of Cardiovascular and Pulmonary Rehabilitation; National Heart, Lung, and Blood Institute; Society for Vascular Nursing; TransAtlantic Inter-Society Consensus; and Vascular Disease Foundation. J Am Coll Cardiol 2006; 47: 1239-312.

11. ElMahdy MF, Kassem HH, Ewis EB, Mahdy SG. Comparison between ultrasound-guided compression and para-aneurysmal saline injection in the treatment of postcatheterization femoral artery pseudoaneurysms. Am J Cardiol 2014; 113: 871-6.

12. Ricci MA, Trevisani GT, Pilcher DB. Vascular complications of 
cardiac catheterization. Am J Surg 1994; 167: 375-8.

13. La Perna L, Olin JW, Goines D, Childs MB, Ouriel K Ultrasound-guided thrombin injection for the treatment of postcatheterization pseudoaneurysms. Circulation 2000; 102: 23915.

14. Zarge J, Villemure P, Mathewson C, Lawrence J, Byars J. Complications related to thrombin injection for pseudoaneurysm repair. J Vasc Tech 2001; 25: 209-12.

15. Sadiq S, Ibrahim W. Thromboembolism complicating thrombin injection of femoral artery pseudoaneurysm: management with intraarterial thrombolysis. J Vasc Interv Radiol 2001; 12: 633-6.
16. Hamraoui K, Ernst SM, van Dessel PF, et al. Efficacy and safety of percutaneous treatment of iatrogenic femoral artery pseudoaneurysm by biodegradable collagen injection. J Am Coll Cardiol 2002; 39: 1297-304.

17. Quarmby JW, Engelke C, Chitolie A, Morgan RA, Belli AM. Autologous thrombin for treatment of pseudoaneurysms. Lancet 2002; 359: 946-7.

18. Chapman WC, Singla N, Genyk Y, et al. A phase 3, randomized, double-blind comparative study of the efficacy and safety of topical recombinant human thrombin and bovine thrombin in surgical hemostasis. J Am Coll Surg 2007; 205: 256-5. 